\title{
PHD3 affects gastric cancer progression by negatively regulating HIF1A
}

\author{
YING-JIE XIA ${ }^{1,2^{*}}$, XIAO-TING JIANG ${ }^{2 *}$, SHI-BIN JIANG ${ }^{2}$, XU-JUN HE $^{2}$, JUN-GANG LUO ${ }^{2}$, \\ ZHENG-CHUANG LIU ${ }^{2}$, LIANG WANG ${ }^{3}$, HOU-QUAN TAO ${ }^{2}$ and JIAN-ZHONG CHEN ${ }^{1}$ \\ ${ }^{1}$ Institute of Immunology, School of Medicine, Zhejiang University, Hangzhou, Zhejiang 310058; \\ ${ }^{2}$ Key Laboratory of Gastroenterology of Zhejiang, Zhejiang Provincial People's Hospital, People's Hospital \\ of Hangzhou Medical College, Hangzhou, Zhejiang 310014; ${ }^{3}$ Department of Surgery, Second Affiliated \\ Hospital, School of Medicine, Zhejiang University, Hangzhou, Zhejiang 310058, P.R. China
}

Received February 22, 2016; Accepted March 14, 2017

DOI: $10.3892 / \mathrm{mmr} .2017 .7455$

\begin{abstract}
Prolyl hydroxylase 3 (PHD3) is widely accepted as a tumor suppressor; however, the expression of PHD3 in various cancer types remains controversial. The present study aimed to investigate the association between PHD3 expression and the clinicopathological features of gastric cancer using reverse transcription-quantitative polymerase chain reaction and immunohistochemistry. The effects of PHD3 in gastric cancer cell lines were assessed using western blot analysis and transwell migration assays. The present results revealed that PHD3 expression was increased in adjacent non-cancerous tissue compared with in gastric cancer tissue, and PHD3 overexpression was correlated with the presence of well-differentiated cancer cells, early cancer stage classification and the absence of lymph node metastasis. In vitro experiments demonstrated that PHD3 may act as a negative regulator of hypoxia-inducible factor- $1 \alpha$ and vascular endothelial growth factor, both of which participate in tumor angiogenesis. In conclusion, the present results suggested that PHD3 may act as a tumor suppressor in gastric cancer. Therefore, the targeted regulation of PHD3 may have potential as a novel therapeutic approach for the treatment of patients with gastric cancer.
\end{abstract}

Correspondence to: Dr Hou-Quan Tao, Key Laboratory of Gastroenterology of Zhejiang, Zhejiang Provincial People's Hospital, People's Hospital of Hangzhou Medical College, 158 Shangtang Road, Hangzhou, Zhejiang 310014, P.R. China

E-mail: taohouquan2008@aliyun.com

Dr Jian-Zhong Chen, Institute of Immunology, School of Medicine, Zhejiang University, 388 Yuhangtang Road, Hangzhou, Zhejiang 310058, P.R. China

E-mail: chenjianzhong@zju.edu.cn

${ }^{*}$ Contributed equally

Key words: prolyl hydroxylase 3, hypoxia-inducible factor- $1 \alpha$, vascular endothelial growth factor, gastric cancer, tumor suppressor

\section{Introduction}

Gastric cancer is the third most common cause of cancer mortality worldwide, accounting for 723,000 deaths in 2012 (1). The low 5 -year survival rate associated with gastric cancer is mainly attributed to the current lack of appropriate biomarkers for early disease diagnosis (2).

Hypoxia can induce angiogenesis, which is a critical step in the generation of tumor vasculature. Therefore, hypoxia in the tumor microenvironment may be a crucial factor for promoting the progression of several types of cancer, including gastric tumors (3). Hypoxia-inducible factor-1 $\alpha$ (HIF1A) is a regulatory factor critical for the adaptation of tumor cells to a hypoxic environment $(4,5)$, and its overexpression can promote tumor growth and metastasis through inducing tumor angiogenesis $(6,7)$. The expression of HIF1 A appears to be potentiated in numerous types of human cancer, including colorectal (8) and edometrioid carcinomas (9), breast (10), pancreatic (11), prostate (12) and ovarian cancer (13), and many more $(14,15)$.

Prolyl hydroxylases (PHDs) form a small protein family with a wide distribution across human tissues, and are considered latent anti-oncogenes (16). PHD3, which is also known as Egl-9 family hypoxia inducible factor 3 , is an important member of the PHD family. PHD3 has been reported to negatively regulate the expression of HIF1A through the degradation of oxygen-dependent proteasomes under hypoxic conditions (17). It has previously been suggested that PHD3 is associated with the local tissue hypoxia that accompanies tumorigenesis, and may act as a tumor suppressor (18). Chen et al (19) demonstrated that PHD3 protected the intestinal epithelial barrier from ulcerative injury, which is one of the causes leading to the development of gastric cancer. However, although the actions of PHD3 in the suppression of angiogenesis, growth and differentiation of tumor cells have previously been reported (20), the detailed molecular mechanisms implicating PHD3 in gastric cancer have yet to be elucidated.

The present study aimed to explore the association between PHD3 expression and the clinicopathological features of gastric cancer, and investigate the biological roles of PHD3 in gastric tumor angiogenesis, invasion and metastasis. 


\section{Materials and methods}

Tissue specimens. Cancerous and adjacent non-cancerous tissue samples were obtained from 70 patients (46 male and 24 female; age, 32-76 years) with gastric cancer who underwent radical gastrectomy at the Zhejiang Provincial People's Hospital (Hangzhou, China) between January 2008 and December 2009. All tissue samples were resectioned and immediately stored at $-80^{\circ} \mathrm{C}$ or fixed in $10 \%$ buffered formalin for further use. The tissue samples of 70 cases were used for immunohistochemistry, 50 cases were used for reverse transcription-quantitative polymerase chain reaction (RT-qPCR) analysis and 9 cases were used for western blot analysis. Routine chemotherapy and radiotherapy were not administered to the patients prior to surgery. The present study was approved by the Ethics Committee of the Zhejiang Provincial People's Hospital. Written informed consent was obtained from the patients.

$R T$ - $q P C R$. Total RNA was extracted from pulverized tissue samples using TRIzol ${ }^{\circledR}$ reagent (Invitrogen; Thermo Fisher Scientific, Inc., Waltham, MA, USA), according to the manufacturer's protocol. Total RNA was reverse transcribed into cDNA using the PrimeScript 1st Strand cDNA Synthesis kit (Takara Bio, Inc., Otsu, Japan). Template RNA solution was heated at $65^{\circ} \mathrm{C}$ for $5 \mathrm{~min}$, then immediately cooled on ice. The $20-\mu 1$ reaction mixture was incubated at $42^{\circ} \mathrm{C}$ for $1 \mathrm{~h}$ and $70^{\circ} \mathrm{C}$ for $15 \mathrm{~min}$, and placed on ice for $2 \mathrm{~min}$. qPCR analysis was performed according to the manufacturer's protocol of SYBR Premix Ex Taq ${ }^{\mathrm{TM}}$ II (Takara Bio, Inc.). The candidate primers are listed in Table I. GAPDH was used as an internal control. Initial denaturation $\left(4 \mathrm{~min}\right.$ at $95^{\circ} \mathrm{C}$ ) was followed by 40 cycles of amplification at $95^{\circ} \mathrm{C}$ for $10 \mathrm{sec}$, annealing at $56^{\circ} \mathrm{C}$ for $30 \mathrm{sec}$, and $72^{\circ} \mathrm{C}$ for $30 \mathrm{sec}$. Melting curve analysis was performed at the end of the PCR cycles. The relative expression levels were calculated using the $2^{-\Delta \Delta \mathrm{Cq}}$ method (21).

Western blot analysis. Pulverized tissue samples were homogenized in lysis buffer (Beyotime Institute of Biotechnology, Haimen, China). Suspensions were centrifuged at 14,000 x g for $5 \mathrm{~min}$ at $4^{\circ} \mathrm{C}$, and the supernatants were collected. The total protein concentrations were detected using an Enhanced Bicinchoninic Acid Protein Assay kit (Beyotime Institute of Biotechnology). Equal amounts of protein $(40 \mu \mathrm{g})$ were separated by SDS-PAGE on a $10 \%$ gel and transferred onto polyvinylidene difluoride membranes (Bio-Rad Laboratories, Inc., Hercules, CA, USA). Membranes were blocked with $5 \%$ non-fat dry milk in Tris-buffered saline for $2 \mathrm{~h}$ at room temperature. Subsequently, membranes were incubated with rabbit anti-human PHD3 (cat. no. ab30782) and HIF1A (cat. no. ab51608; both Abcam, Cambridge, UK), vascular endothelial growth factor (VEGF; cat. no. 19003-1-AP) and GAPDH (cat. no. 10494-1-AP; both ProteinTech Group, Inc., Chicago, IL, USA) primary antibodies overnight at $4^{\circ} \mathrm{C}$. Following washing with TBS-Tween 20 , membranes were incubated with the secondary antibody (goat anti-rabbit immunoglobulin G, horseradish peroxidase-conjugated; cat. no. HA1001-100; Hangzhou HuaAn Biotechnology Co., Ltd., Hangzhou, China) at room temperature for $2 \mathrm{~h}$, then washed and incubated for 1 min with an enhanced chemiluminescence kit (Beyotime
Institute of Biotechnology) to visualize the bands. GAPDH was used as a loading control. The blots were quantified using the FluorChem FC2 imaging system (ProteinSimple, San Jose, CA, USA).

Cell culture. Human gastric epithelial GES-1 cells and the human gastric cancer cell lines SGC7901, BGC823, MKN45, MKN28, BGC803 and AGS were purchased from the Cell Bank of the Shanghai Institute of Biochemistry and Cell Biology (Shanghai, China). Cells were cultured in RPMI-1640 medium (HyClone; GE Healthcare Life Sciences, Logan, UT, USA) supplemented with $10 \%$ fetal bovine serum (Sijiqing; Zhejiang Tianhang Biotechnology Co., Ltd., Hangzhou, China), $100 \mathrm{U} / \mathrm{ml}$ streptomycin and $100 \mathrm{U} / \mathrm{ml}$ penicillin, and maintained at $37^{\circ} \mathrm{C}$ in a $5 \% \mathrm{CO}_{2}$ atmosphere. Cells were passaged at $80 \%$ confluency using $1 \mathrm{mmol} / 1$ EDTA- $0.025 \%$ trypsin for 1-3 $\mathrm{min}$. The results of the RT-qPCR analysis demonstrated that AGS cells exhibited the lowest PHD3 expression and MKN28 cells exhibited the highest expression among the gastric cancer cells. It is necessary to note that the MKN28 cell line is known to be mis-identified; it is derived from the MKN47 cell line.

PHD3 transfection. Gastric cancer AGS and MKN28 cells were plated at a density of $5.0 \times 10^{5}$ cells/well in 6 -well dishes $24 \mathrm{~h}$ prior to transfection. In order to upregulate the expression of PHD3, $1 \mu \mathrm{g}$ total RNA was extracted from GES-1 cell lines with TRIzol ${ }^{\circledR}$ reagent (Invitrogen; Thermo Fisher Scientific, Inc.), and was used to synthesize full-length PHD3 CDNAs with Taq Master Mix (Novoprotein; Sinobio Chemistry Co., Ltd., Dalian, China), according to the manufacturer's protocol. The primers, containing Hind III and XhoI restriction enzyme cutting sites, are listed in Table I. PCR-amplified full-length human PHD3 cDNA was cloned into pcDNA3.1 (pcDNA3.1-PHD3), and pcDNA3.1 (Invitrogen; Thermo Fisher Scientific, Inc.) was used as negative control (pcDNA3.1-NC). pcDNA3.1-PHD3 and pcDNA3.1-NC were transfected into AGS cells. The sequence of PHD3 short hairpin (sh)RNA DNA (shRNA-PHD3; Merck KGaA, Darmstadt, Germany) was CCGGCTACGTCA AGGAGAGGTCTA ACTCGA GTTAGACCTCTCCTTGACGTAGTTTTT. The negative control (shRNA-NC; GACTTCATAAGGCGCATGC), whose sequence was not isogeneous with any human gene, and shRNA-PHD3 were cloned into the pYr-1.1 vector (Yinrun Biotechnology, Inc., Changsha, China), and then transfected into MKN28 cells. Transfection was performed using Lipofectamine 2000 (Invitrogen; Thermo Fisher Scientific, Inc.) as the delivery agent, according to the manufacturer's protocol.

Transwell assays. The migratory and invasive capabilities of transfected cells were assessed. Transwell migration assay was carried out in 24-well plates using the Costar Transwell assay kit (cat no. 3422; Corning Incorporated, Corning, NY, USA). The invasion assay was carried out using invasion chambers (cat no. 354480; BD Biosciences, San Jose, CA, USA) pre-coated with Matrigel. Cells were seeded in the upper chamber at a density of $2.0 \times 10^{5}$ cells/well, and $30 \%$ FBS RPMI-1640 culture medium was added to the lower chamber. Following $48 \mathrm{~h}$ of incubation at $37^{\circ} \mathrm{C}$ in a $5 \% \mathrm{CO}_{2}$ atmosphere, 
Table I. Primer sequence table.

\begin{tabular}{llc}
\hline Primer & \multicolumn{1}{c}{ Sequence, 5'-3' $^{\prime}$} & Temperature, ${ }^{\circ} \mathrm{C}$ \\
\hline PHD3 & F: TTGCCAGATGAAGTTATTTGCT & 56 \\
& R: TTCCCTCGCTGTGCTCCT & 56 \\
HIF1A & F: TCTCCATCTCCTACCCACATACAT & 56 \\
& R: TGCTCTGTTTGGTAGGCTGT & 56 \\
VEGF & F: GGCTCTGACCAGGAGTTTG & 56 \\
GAPDH & R: CAACAATGTGTCTCTTCTCTTCG & 56 \\
FHD3 cDNA & R: CTGAGGTCGGAGTCAACGG & 61 \\
& F: CCCAAGCTTGATGCCCCTGGGACACATCAT & \\
\hline
\end{tabular}

PHD, prolyl hydroxylase; HIF1A, hypoxia-inducible factor-1 $\alpha$; VEGF, vascular endothelial growth factor.

unmigrated or noninvasive cells were removed from the upper surface of the transwell membranes using a cotton swab, and the migrated or invaded cells on the lower membrane surface were fixed with ethyl alcohol for $15 \mathrm{~min}$ at room temperature, stained with hematoxylin for $5 \mathrm{~min}$ at room temperature and counted under a light microscope (Olympus Corporation, Tokyo, Japan).

Immunohistochemistry. Tissues were fixed in $10 \%$ neutral buffered formalin for one day at room temperature, dehydrated by gradient ethanol $(75,85,95 \%$ and absolute ethyl alcohol), cleared with absolute xylene and embedded in paraffin. Tissue sections were deparaffinized and rehydrated. Heat-induced antigen retrieval was carried out in $0.01 \mathrm{M}$ citrate buffer. Sections were incubated with $3 \% \mathrm{H}_{2} \mathrm{O}_{2}$ for $20 \mathrm{~min}$ at room temperature and blocked with normal goat serum (Invitrogen; Thermo Fisher Scientific, Inc.). They were subsequently incubated with rabbit anti-human PHD3 primary antibody (1:250 in PBS; cat. no. ab30782; Abcam) overnight at $4^{\circ} \mathrm{C}$, followed by incubation with biotinylated secondary antibody at room temperature for $20 \mathrm{~min}$, and then with horseradish peroxidase-conjugated polymer (Histostain-Plus kit; cat. no. 859043; Invitrogen; Thermo Fisher Scientific, Inc.) at room temperature for $20 \mathrm{~min}$ according to the manufacturer's protocol. Finally, the sections were stained with diaminobenzidine (DAB Substrate kit; Wuhan Boster Biological Technology, Ltd., Wuhan, China), lightly counterstained with hematoxylin for $5 \mathrm{~min}$ at room temperature and mounted.

Based on the proportion and the intensity of positively stained cells, the degree of immunostaining was reviewed under a light microscope (5 fields; magnification, x200) and scored independently by two observers blinded to the clinical outcomes. The staining intensity was graded on a scale between 0 and $3+(0$, no staining; $1+$, weak immunoreactivity; $2+$, moderate immunoreactivity; $3+$, strong immunoreactivity). The percentage of cells that exhibited positive PHD3 staining was scored as follows: $1,0-25 \%$ positive cells; $2,26-50 \%$ positive; $3,51-75 \%$ positive; $4,76-100 \%$ positive. The staining intensity score and the $\%$ immunoreactivity score were then multiplied to obtain a composite score. The values of the composite score ranged between 0 and 12 , with $0-5$ defined as low expression, and $\geq 6$ defined as high expression.

Statistical analysis. Comparisons between groups were analyzed using paired samples t tests. Survival curves were estimated using the Kaplan-Meier method. Cox regression analysis was used to assess the prognostic values. $\mathrm{P}<0.05$ was considered to indicate a statistically significant difference. The analysis was performed using SPSS software version 13.0 (SPSS, Inc., Chicago, IL, USA).

\section{Results}

Expression of PHD3 in gastric cancer and adjacent non-cancerous tissue. The results of RT-qPCR revealed that 43 out of $50(86 \%)$ paired samples exhibited reduced PHD3 mRNA expression in cancerous tissue compared with in adjacent non-cancerous tissue, as analyzed by paired samples t-test (Fig. 1A and B; P<0.001). Results from western blot analysis and immunohistochemistry were consistent with RT-qPCR. In addition, PHD3 expression was decreased in gastric cancer cells, compared with the expression of PHD3 in gastric epithelial GES-1 cells (Fig. 1C). Western blot analysis revealed that $88.9 \%$ (8/9 cases) of samples from adjacent non-cancerous tissue exhibited increased PHD3 expression compared with in paired cancer tissue samples (Fig. 2). Immunohistochemistry demonstrated that PHD3 could be detected in all adjacent non-cancerous tissues; 59 out of 70 $(84.3 \%)$ paired samples exhibited increased PHD3 expression in adjacent non-cancerous tissue compared with in cancerous tissue (Fig. 3).

Association between PHD3 and clinicopathological features of gastric cancer. Immunohistochemistry results demonstrated that PHD3 was mainly localized in the cytoplasm and seldom in the nucleus (Fig. 3). Correlation analysis revealed PHD3 to be associated with tumor differentiation $(\mathrm{P}=0.013)$, TNM stage $(\mathrm{P}=0.031)$ and lymph node metastasis $(\mathrm{P}=0.014)$, but not with age $(\mathrm{P}=0.853)$ or gender $(\mathrm{P}=0.851)$ of the patients, or with tumor size $(\mathrm{P}=0.216)$ (Table II). 

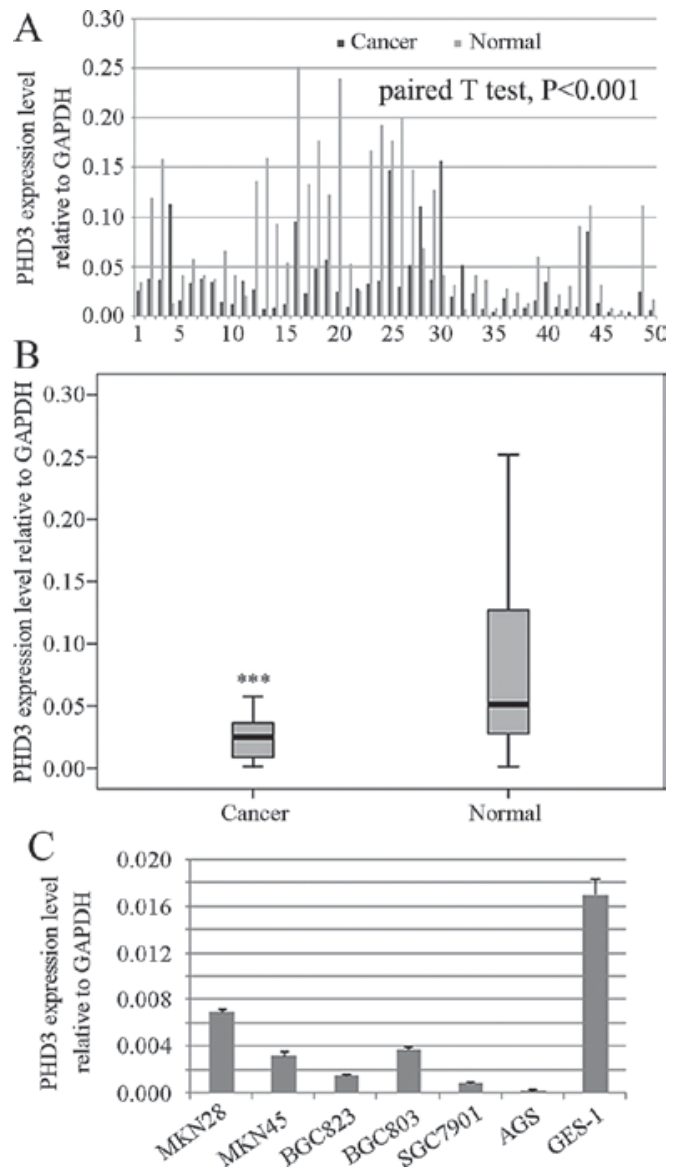

Figure 1. PHD3 mRNA expression levels were assessed using reverse transcription-quantitative polymerase chain reaction. (A) PHD3 mRNA expression levels in 50 paired samples from gastric cancer and adjacent non-cancerous tissues. Each sample was analyzed in triplicate and normalized to GAPDH. PHD3 mRNA expression levels were lower in 43 gastric cancer tissue samples compared with in their pair-matched samples from adjacent non-cancerous tissue. (B) PHD3 mRNA expression levels in 50 paired samples from gastric cancer and adjacent non-cancerous tissue presented in boxplot. ${ }^{* * *} \mathrm{P}<0.001$ vs. Normal. (C) PHD3 mRNA expression levels in human gastric epithelial GES-1 cells and human gastric cancer cell lines (SGC7901, BGC823, MKN45, MKN28, BGC803 and AGS). Relative PHD3 mRNA expression levels in gastric cancer cell lines were decreased compared with in GES-1 cells. Among the gastric cancer cell lines, AGS cells exhibited the lowest PHD3 expression, whereas MKN28 cells exhibited the highest PHD3 expression. Relative expression of PHD3 was normalized to the endogenous control GAPDH. Each sample was analyzed in triplicate. PHD, prolyl hydroxylase.

The detection rate for increased PHD3 expression was $64 \%(16 / 25)$ in well and moderately differentiated specimens, which was higher compared with in poorly differentiated or undifferentiated specimens $(33.3 \%, 15 / 45)$. High PHD3 expression was detected in $22.7 \%(5 / 22)$ of gastric cancer specimens with lymph node metastasis, which was lower than in specimens without lymph node metastasis $(54.2 \%, 26 / 48)$. The detection rate of high PHD3 expression was also increased in early TNM stages $(54.8 \%$ in $<$ IIb stage vs. $28.6 \%$ in $\geq \mathrm{IIb}$ stage). The 5-year survival rate of patients with high PHD3 expression $(64.5 \%)$ was higher than those with low PHD3 expression (41.0\%). Survival curves were estimated using the Kaplan-Meier method (Fig. 4, $\mathrm{P}=0.063$ ).

PHD3 transfection of gastric cancer cell lines. The results of the RT-qPCR analysis demonstrated that PHD3 protein
A
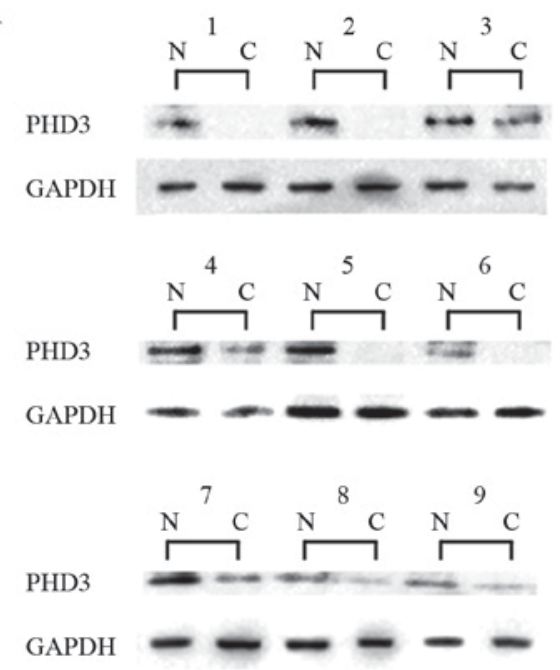

B

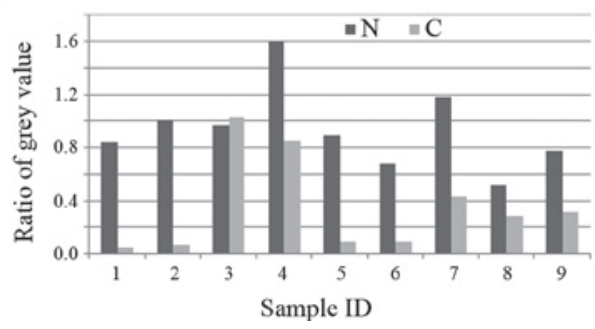

Figure 2. Protein lysates obtained from tissue samples were analyzed using western blotting. (A) GAPDH served as an internal control. PHD3 protein expression was assessed in 9 paired samples from gastric cancer and adjacent non-cancerous tissues. PHD, prolyl hydroxylase; $\mathrm{N}$, adjacent non-cancerous tissue sample; $\mathrm{C}$, gastric cancer tissue sample. (B) The ratio of the grey value in each sample/the grey value of internal reference GAPDH.

expression levels appeared lower in AGS cells compared with in other gastric cancer cell lines (Fig. 1C); however, PHD3 expression increased after AGS cells were transfected with pcDNA3.1-PHD3 (Fig. 5). Conversely, PHD3 expression levels were decreased in MKN28 cells following transfection with shRNA-PHD3 (Fig. 5). Furthermore, HIF1A and VEGF expression levels appeared decreased following PHD3 upregulation in AGS cells, and increased following PHD3 downregulation in MKN28 cells (Fig. 5).

The in vitro transwell migration and invasion assays revealed that AGS cells transfected with pcDNA3.1-PHD3 exhibited decreased migratory and invasive capabilities compared with negative control cells. Conversely, MKN28 cells transfected with shRNA-PHD3 exhibited increased migratory and invasive capabilities compared with negative control cells (Fig. 6).

\section{Discussion}

Previous studies have revealed that the protein expression levels of PHD3 are low in breast and colon cancer $(22,23)$, whereas they appear high in lung (24) and pancreatic cancer (25). However, the mechanisms underlying the implication of PHD3 in gastric cancer have yet to be elucidated. The present study demonstrated that PHD3 levels are markedly decreased in gastric cancer tissue compared with in adjacent non-cancerous tissue. These 

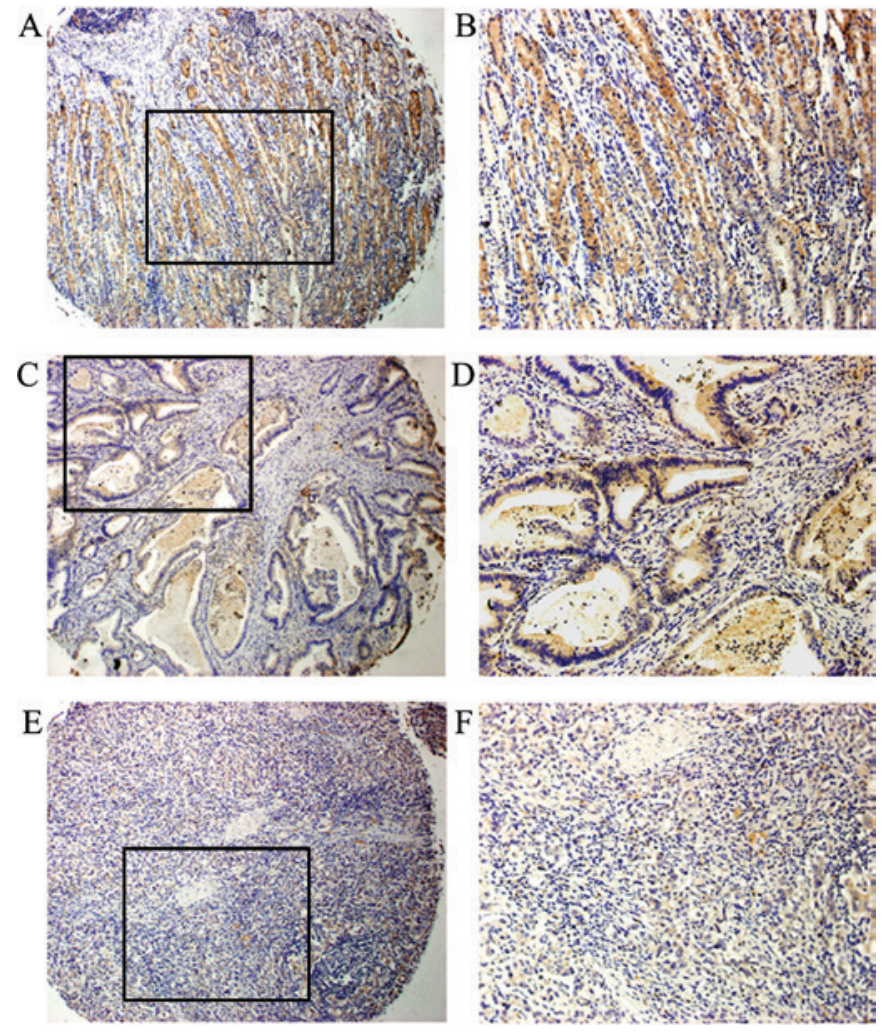

Figure 3. PHD3 expression was assessed using immunohistochemistry. (A) Sample from non-cancerous tissue adjacent to gastric cancer tissue (100x magnification). (B) 200x magnification of marked area in panel A. (C) Tissue sample from moderately/well-differentiated adenocarcinoma (100x magnification). (D) 200x magnification of marked area in panel C. (E) Tissue sample from undifferentiated/poorly-differentiated adenocarcinoma (100x magnification). (F) 200x magnification of marked area in panel E. Yellow-brown granules, mainly in the cytoplasm and seldom in the nucleus, indicate the presence of PHD3. PHD, prolyl hydroxylase.

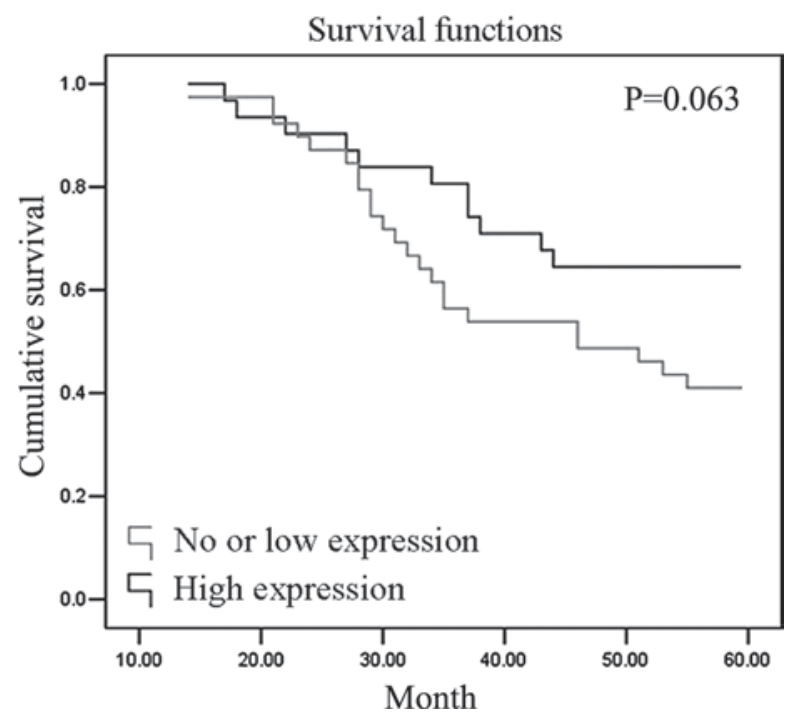

Figure 4. Kaplan-Meier survival curves from 70 patients with gastric cancer, differentiated by initial PHD3 expression level. PHD, prolyl hydroxylase.

results suggested that PHD3 may act as a tumor suppressor (26). PHD3 expression levels may vary in different tissue types, and according to the degree of tumor differentiation.
A

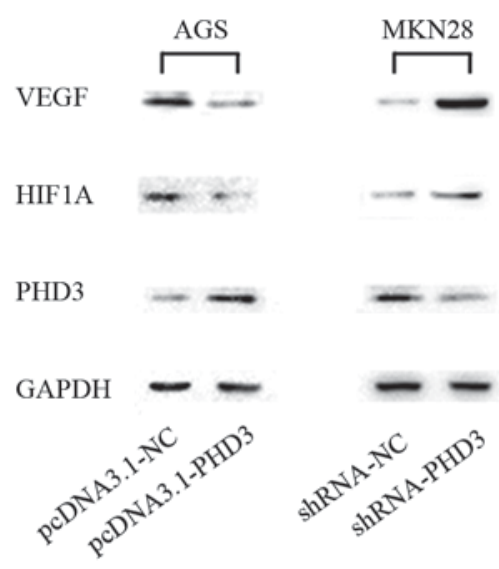

B
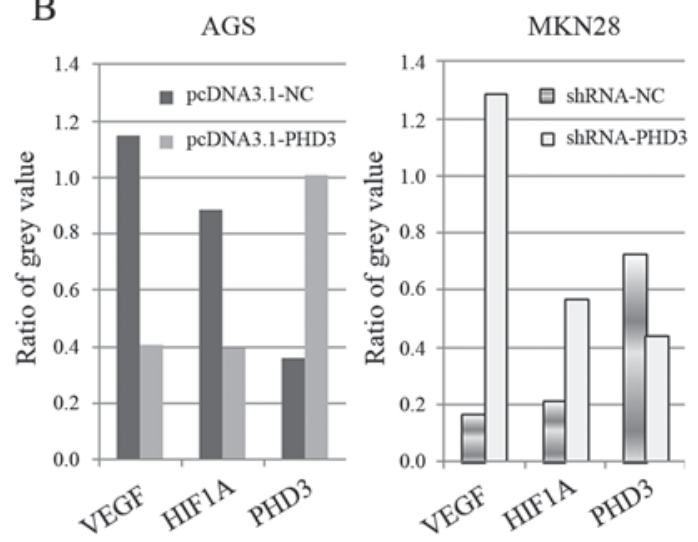

Figure 5. Protein lysates obtained from cultured cells were analyzed using western blotting. (A) GAPDH served as an internal control. Protein expression levels of PHD3, HIF1A and VEGF in AGS and MKN28 gastric cancer cells. AGS cells were transfected with pcDNA3.1-PHD3 and MKN28 cells were transfected with shRNA-PHD3. PHD, prolyl hydroxylase; HIF1A, hypoxia-inducible factor-1 $\alpha$; VEGF, vascular endothelial growth factor; shRNA, short hairpin RNA; NC, negative control. (B) The ratio of the grey value in each group/the grey value of internal reference GAPDH.

Correlation analysis of PHD3 expression with regards to the clinicopathological features of gastric cancer revealed that increased PHD3 expression was correlated with a high degree of differentiation of cancer cells, early TNM stage and decreased lymph node metastasis. These results are consistent with previous studies $(20,27)$ and suggest that PHD3, or a putative protein target downstream of PHD3, may participate in tumor progression. Su et al (25) reported that PHD3 was upregulated in well-differentiated specimens of pancreatic cancer, whereas undifferentiated tumors exhibited reduced PHD3 expression. Although the survival rate of gastric cancer patients with high PHD3 expression appeared higher than that of patients with low PHD3 expression, survival analysis revealed the difference to be insignificant. It has previously been suggested that PHD3 expression may be used as a prognostic marker for disease-specific survival in several types of tumors $(23,28-30)$. Further studies, using larger sample sizes, are required to elucidate the association between PHD3 expression and the prognosis of gastric cancer.

To explore the mechanism underlying the implication of PHD3 in gastric cancer, PHD3 expression was assessed in gastric cancer cell lines. All gastric cancer cell lines exhibited lower PHD3 protein expression levels compared with the human 
Table II. Association between PHD3 expression and clinical parameters of gastric cancer.

\begin{tabular}{|c|c|c|c|}
\hline \multirow[b]{2}{*}{ Clinical parameter } & \multicolumn{2}{|c|}{ PHD3 expression (\%) } & \multirow[b]{2}{*}{ P-value (2-sided) } \\
\hline & Low & High & \\
\hline Age, years & & & 0.853 \\
\hline$<61$ & $18(54.5)$ & $15(45.5)$ & \\
\hline$\geq 61$ & $21(56.7)$ & $16(43.3)$ & \\
\hline Gender & & & 0.851 \\
\hline Female & $13(54.2)$ & $11(45.8)$ & \\
\hline Male & $26(56.5)$ & $20(43.5)$ & \\
\hline Size, cm & & & 0.216 \\
\hline$<5$ & $27(61.3)$ & $17(38.7)$ & \\
\hline$\geq 5$ & $12(46.2)$ & $14(53.8)$ & \\
\hline Differentiation & & & 0.013 \\
\hline Moderate and well & $9(36)$ & $16(64)$ & \\
\hline Poor or non-differentiated & $30(66.7)$ & $15(33.3)$ & \\
\hline TNM stages & & & 0.031 \\
\hline <IIb stage & $19(45.2)$ & $23(54.8)$ & \\
\hline$\geq$ IIlb stage & $20(71.4)$ & 8 (28.6) & \\
\hline Lymph node metastasis & & & 0.014 \\
\hline No & $22(45.8)$ & $26(54.2)$ & \\
\hline Yes & $17(77.3)$ & $5(22.7)$ & \\
\hline
\end{tabular}

PHD, prolyl hydroxylase.

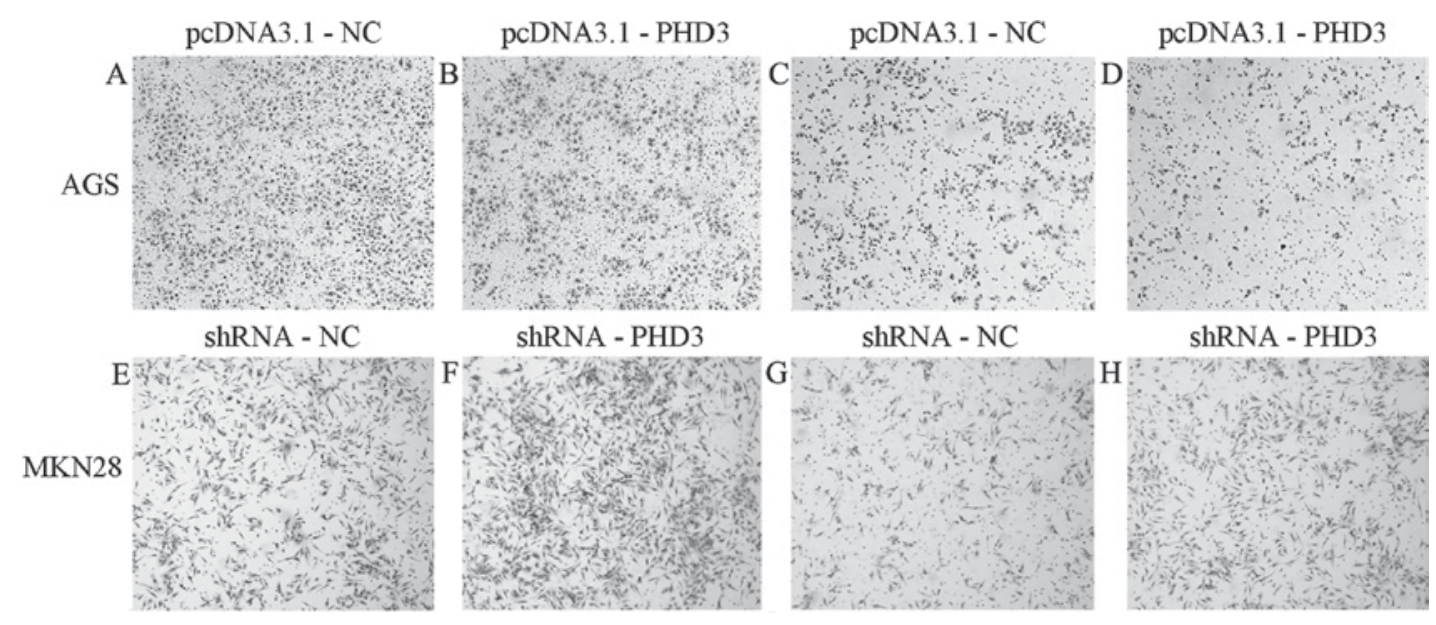

Figure 6. Migration and invasion assays in AGS and MKN28 gastric cancer cells. AGS cells were transfected with pcDNA3.1-PHD3 and exhibited impaired (A and B) migratory and (C and D) invasive capabilities compared with NC cells. MKN28 cells were transfected with shRNA-PHD3 and exhibited increased (E and F) migratory and ( $\mathrm{G}$ and $\mathrm{H}$ ) invasive capabilities compared with NC cells. PHD, prolyl hydroxylase; shRNA, short hairpin RNA; NC, negative control.

gastric epithelial cell line GES-1. These results are consistent with the present findings in gastric cancer tissue samples, as well as with the findings reported by Tanaka et al (31).

To investigate the effects of PHD3 on the migratory and invasive capabilities of gastric cancer cells, PHD3 expression was silenced in MKN28 cells, which resulted in increased cellular migration and invasion. Conversely, upregulation of PHD3 expression in AGS cells impaired their migratory and invasive capabilities. PHD3 upregulation has also been reported to inhibit the migration and invasion of pancreatic cancer cells (25). In order to investigate the mechanism underlying the actions of PHD3 on cellular migration and invasion, the expression levels of HIF1A and VEGF were assessed in AGS and MKN28 gastric cancer cells. Knockdown of PHD3 in MKN28 cells increased HIF1A and VEGF expression, whereas upregulation of PHD3 expression in AGS cells decreased the expression of HIF1A and VEGF. These results suggested that PHD3 may negatively regulate the expression of HIF1A. Furthermore, HIF1A has been reported to participate in tumor growth and metastasis, through the induction of VEGF. The 
present results are consistent with previous studies $(32,33)$, which reported that decreased HIF1A expression is associated with impaired angiogenic activity. Research on the biological functions of HIF1A (34) proposed a similar view. Since the formation of tumor vasculature is critical for tumor progression, it may be hypothesized that PHD3 can interfere in the angiogenic processes of gastric tumors, through the regulation of HIF1A expression.

In conclusion, the present study suggested that PHD3 may serve a tumor-suppressing role in gastric cancer. PHD3 overexpression may reduce the migratory and invasive capacity of gastric cancer cells, and inhibit the formation of tumor vasculature via negatively regulating HIF1A, which has been revealed to control VEGF transcription. These results demonstrate the potential for the development of novel therapeutic strategies for the treatment of gastric cancer, based on the targeted regulation of PHD3.

\section{Acknowledgements}

The present study was supported by the Medicine and Health Research Foundation of Zhejiang Province (grant nos. 2012KYB016 and 2011KYA069), the National Key Basic Research Program of China (grant no. 2014CB542101) and the Zhejiang Provincial Natural Science Foundation of China (grant no. LY14H100003).

\section{References}

1. Ferlay J, Soerjomataram I, Dikshit R, Eser S, Mathers C, Rebelo M, Parkin DM, Forman D and Bray F: Cancer incidence and mortality worldwide: Sources, methods and major patterns in GLOBOCAN 2012. Int J Cancer 136: E359-E386, 2015.

2. Baniak N, Senger JL, Ahmed S, Kanthan SC and Kanthan R: Gastric biomarkers: A global review. World J Surg Oncol 14: 212, 2016.

3. Muz B, de la Puente P, Azab F and Azab AK: The role of hypoxia in cancer progression, angiogenesis, metastasis, and resistance to therapy. Hypoxia (Auckl) 3: 83-92, 2015.

4. Guo X, Li D, Chen Y, An J, Wang K, Xu Z, Chen Z and Xing J: SNP rs2057482 in HIF1A gene predicts clinical outcome of aggressive hepatocellular carcinoma patients after surgery. Sci Rep 5: 11846, 2015.

5. Fraga A, Ribeiro R, Principe P, Lobato C, Pina F, Maurício J, Monteiro C, Sousa H, Calais da Silva F, Lopes C and Medeiros R: The HIF1A functional genetic polymorphism at locus +1772 associates with progression to metastatic prostate cancer and refractoriness to hormonal castration. Eur J Cancer 50: 359-365, 2014.

6. Shyu KG, Hsu FL, Wang MJ, Wang BW and Lin S: Hypoxia-inducible factor 1alpha regulates lung adenocarcinoma cell invasion. Exp Cell Res 313: 1181-1191, 2007.

7. Zhang W, Zhang H and Xing L: Antisense oligonucleotide of hypoxia-inducible factor-1alpha suppresses growth and tumorigenicity of lung cancer cells A549. J Huazhong Univ Sci Technolog Med Sci 26: 448-450, 2006.

8. Baba Y, Nosho K, Shima K, Irahara N, Chan AT, Meyerhardt JA, Chung DC, Giovannucci EL, Fuchs CS and Ogino S: HIF1A overexpression is associated with poor prognosis in a cohort of 731 colorectal cancers. Am J Pathol 176: 2292-2301, 2010.

9. Espinosa I, José Carnicer M, Catasus L, Canet B, D'angelo E, Zannoni GF and Prat J: Myometrial invasion and lymph node metastasis in endometrioid carcinomas: Tumor-associated macrophages, microvessel density, and HIF1A have a crucial role. Am J Surg Pathol 34: 1708-1714, 2010.

10. Deb S, Johansson I, Byrne D, Nilsson C, Investigators $k$ Constable L, Fjällskog ML, Dobrovic A, Hedenfalk I and Fox S: Nuclear HIF1A expression is strongly prognostic in sporadic but not familial male breast cancer. Mod Pathol 27: 1223-1230, 2014.
11. Hoffmann AC, Mori R, Vallbohmer D, Brabender J, Klein E, Drebber U, Baldus SE, Cooc J, Azuma M, Metzger R, et al: High expression of HIFla is a predictor of clinical outcome in patients with pancreatic ductal adenocarcinomas and correlated to PDGFA, VEGF, and bFGF. Neoplasia 10: 674-679, 2008.

12. Vainrib M, Golan M, Amir S, Dang DT, Dang LH, Bar-Shira A, Orr-Urtreger A, Matzkin H and Mabjeesh NJ: HIF1A C1772T polymorphism leads to HIF- $1 \alpha$ mRNA overexpression in prostate cancer patients. Cancer Biol Ther 13: 720-726, 2012.

13. Nakai $H$, Watanabe $Y$, Ueda $H$ and Hoshiai $H$ : Hypoxia inducible factor 1-alpha expression as a factor predictive of efficacy of taxane/platinum chemotherapy in advanced primary epithelial ovarian cancer. Cancer Lett 251: 164-167, 2007.

14. Zhong H, De Marzo AM, Laughner E, Lim M, Hilton DA, Zagzag D, Buechler P, Isaacs WB, Semenza GL and Simons JW: Overexpression of hypoxia-inducible factor 1alpha in common human cancers and their metastases. Cancer Res 59: 5830-5835, 1999.

15. Talks KL, Turley H, Gatter KC, Maxwell PH, Pugh CW, Ratcliffe PJ and Harris AL: The expression and distribution of the hypoxia-inducible factors HIF-1alpha and HIF-2alpha in normal human tissues, cancers, and tumor-associated macrophages. Am J Pathol 157: 411-421, 2000.

16. Minervini G, Quaglia F and Tosatto SC: Insights into the proline hydroxylase (PHD) family, molecular evolution and its impact on human health. Biochimie 116: 114-124, 2015.

17. Place TL, Fitzgerald MP, Venkataraman S, Vorrink SU, Case AJ, Teoh ML and Domann FE: Aberrant promoter CpG methylation is a mechanism for impaired PHD3 expression in a diverse set of malignant cells. PLoS One 6: e14617, 2011.

18. Zhou Y, Liang QL, Ou WT, Liu QL, Zhang XN, Li ZY and Huang X: Effect of stable transfection with PHD3 on growth and proliferation of HepG2 cells in vitro and in vivo. Int J Clin Exp Med 7: 2197-2203, 2014.

19. Chen Y, Zhang HS, Fong GH, Xi QL, Wu GH, Bai CG, Ling ZQ, Fan L, Xu YM, Qin YQ, et al: PHD3 Stabilizes the Tight Junction Protein Occludin and Protects Intestinal Epithelial Barrier Function. J Biol Chem 290: 20580-20589, 2015.

20. Liu QL, Liang QL, Li ZY, Zhou Y, Ou WT and Huang ZG: Function and expression of prolyl hydroxylase 3 in cancers. Arch Med Sci 9: 589-593, 2013.

21. Livak KJ and Schmittgen TD: Analysis of relative gene expression data using real-time quantitative PCR and the 2(-Delta Delta C(T)) Method. Methods 25: 402-408, 2001.

22. Rawluszko AA, Bujnicka KE, Horbacka K, Krokowicz P and Jagodzinski PP: Expression and DNA methylation levels of prolyl hydroxylases PHD1, PHD2, PHD3 and asparaginyl hydroxylase FIH in colorectal cancer. BMC Cancer 13: 526, 2013.

23. Peurala E, Koivunen P, Bloigu R, Haapasaari KM and Jukkola-Vuorinen A: Expressions of individual PHDs associate with good prognostic factors and increased proliferation in breast cancer patients. Breast Cancer Res Treat 133: 179-188, 2012.

24. Chen S, Zhang J, Li X, Luo X, Fang J and Chen H: The expression of prolyl hydroxylase domain enzymes are up-regulated and negatively correlated with Bcl-2 in non-small cell lung cancer. Mol Cell Biochem 358: 257-263, 2011.

25. Su Y, Loos M, Giese N, Hines OJ, Diebold I, Görlach A, Metzen E, Pastorekova S, Friess H and Büchler P: PHD3 regulates differentiation, tumour growth and angiogenesis in pancreatic cancer. Br J Cancer 103: 1571-1579, 2010.

26. Tennant DA and Gottlieb E: HIF prolyl hydroxylase-3 mediates alpha-ketoglutarate-induced apoptosis and tumor suppression. J Mol Med (Berl) 88: 839-849, 2010.

27. Xue J, Li X, Jiao S, Wei Y, Wu G and Fang J: Prolyl hydroxylase-3 is down-regulated in colorectal cancer cells and inhibits IKKbeta independent of hydroxylase activity. Gastroenterology 138: 606-615, 2010.

28. Andersen S, Donnem T, Stenvold H, Al-Saad S, Al-Shibli K, Busund LT and Bremnes RM: Overexpression of the HIF hydroxylases PHD1, PHD2, PHD3 and FIH are individually and collectively unfavorable prognosticators for NSCLC survival. PLoS One 6: e23847, 2011.

29. Couvelard A, Deschamps L, Rebours V, Sauvanet A, Gatter K, Pezzella F, Ruszniewski P and Bedossa P: Overexpression of the oxygen sensors PHD-1, PHD-2, PHD-3, and FIH Is associated with tumor aggressiveness in pancreatic endocrine tumors. Clin Cancer Res 14: 6634-6639, 2008. 
30. Gossage L, Zaitoun A, Fareed KR, Turley H, Aloysius M Lobo DN, Harris AL and Madhusudan S: Expression of key hypoxia sensing prolyl-hydroxylases PHD1, -2 and -3 in pancreaticobiliary cancer. Histopathology 56: 908-920, 2010.

31. Tanaka T, Li TS, Urata Y, Goto S, Ono Y, Kawakatsu M, Matsushima H, Hirabaru M, Adachi T, Kitasato A, et al: Increased expression of PHD3 represses the HIF-1 signaling pathway and contributes to poor neovascularization in pancreatic ductal adenocarcinoma. J Gastroenterol 50: 975-983, 2015.

32. Rishi MT, Selvaraju V, Thirunavukkarasu M, Shaikh IA, Takeda K, Fong GH, Palesty JA, Sanchez JA and Maulik N: Deletion of prolyl hydroxylase domain proteins (PHD1, PHD3) stabilizes hypoxia inducible factor-1 alpha, promotes neovascularization and improves perfusion in a murine model of hind-limb ischemia. Microvasc Res 97: 181-188, 2015.
33. Spanberger T, Berghoff AS, Dinhof C, Ilhan-Mutlu A, Magerle M, Hutterer M, Pichler J, Wöhrer A, Hack1 M, Widhalm G, et al: Extent of peritumoral brain edema correlates with prognosis, tumoral growth pattern, HIF1a expression and angiogenic activity in patients with single brain metastases. Clin Exp Metastasis 30: 357-368, 2013.

34. Lee JW, Bae SH, Jeong JW, Kim SH and Kim KW: Hypoxia-inducible factor (HIF-1)alpha: Its protein stability and biological functions. Exp Mol Med 36: 1-12, 2004. 